\title{
Un Libro Sobre Francisco Romero *
}

No es la primera vez que Hugo Rodríguez-Alcalá frecuenta el tema de este libro. Una labor de años y una tenacidad y rigor ejemplares han precedido a esta producción. Nuestro autor, en efecto, había estudiado previamente a Francisco Romero. En I954 publicó, con el auspicio de Columbia University, la primera exposición de conjunto sobre el pensador argentino, con datos biobibliográficos y antología." "Francisco Romero, filósofo de América" y "La crisis contemporánea según Francisco Romero"'2 son, junto con su tesis Francisco Romero, el pensador de la moderna Argentina, otras muestras de su interés por el tema. Pero Rodríguez-A1calá ha hecho más: ha estudiado con igual dedicación el pensamiento de Alejandro Korn. Aunque en nuestros países por regla general, para descubrir la formación de un pensador hay que acudir a las fuentes europeas, los antecedentes vernáculos son, a veces, importantes. La referencia europea puede iluminar las influencias filosóficas determinantes, las preocupaciones temáticas y los supuestos teóricos; pero la referencia nacional puede descubrir una continuidad de magisterio, de clima intelectual, de propósitos prácticos. Así lo es sin duda en el caso de Alejandro Korn y Francisco Romero, iniciador el uno, continuador el otro, con mayores al. cances, de una tradición filosófica en la Argentina. Por eso queríamos llamar la atención sobre la importancia que reviste el profundo conocimiento de la obra de Korn para interpretar la "misión" de Francisco Ro-

* Hugo Rodríguez-Alcalá, Misión y pensamiento de Francisco Romero, Prólogo de José Ferrater Mora. Universidad Nacional Autónoma de México, México, 1959.

1 Francisco Romero. Vida y obra. Bibliografia. Antologia. New York, Hispanic Institute, Columbia University, 1954.

2 Ambos reproducidos en su reciente libro Korn, Romero, Güiraldes, Unamuno, Ortega..., México, 1958. 
mero, que es una de las dos principales tareas que Rodríguez-Alcalá se ha propuesto, como lo revela el título de su libro. ${ }^{3}$

El primer capítulo do Misión y pensamiento de Francisco Romero expone el clima positivista, sobre cuyo trasfondo se destacarán las ideas centrales del pensador argentino. En rigor, y así lo advierte el autor al comienzo, "se exponen las ideas de Francisco Romero acerca del origen y desarrollo del positivismo" (p. rI). Acertadamente distingue entonces el autor dos aspectos: a) el positivismo como capítulo de la historia de las ideas en Argentina y, por tanto, ambiente que rodea a la formación filosófica de Romero; y b) las ideas de Romero sobre el positivismo euro. peo. En cuanto al primer aspecto, Rodríguez-Alcalá señala la existencia del llamado "positivismo autóctono", que representa eminentemente Alberdi (en esto sigue no sólo a Romero, sino también a Korn, de quien es originariamente la idea), y luego expone la actuación de los pedagogos comtianos - Alfredo Forreira ante todo-y de los cientificistas, en particular Ingenieros. Quizá alguien encuentre objetable el uso exclusivo de los puntos de vista de Romero para describir este período de la historia ideológica argentina. Sin embargo, deben tenerse en cuenta dos factores: por un lado, el natural interés que estas opiniones revisten para quien está exponiendo la obra de Romero; por otro, el hecho de que no abunden las obras generales sobre el asunto, única fuente a la que podría recurrir el autor, pues de otro modo se habría convertido en investigador del positivismo argentino, a lo cual, naturalmente, no estaba obligado. En el examen del positivismo europeo ara de rigor, en cambio, el análisis de la interpretación de Romero. Este ha estudiado repetidamente y desde distintos ángulos el contenido del positivismo. Lo ha hecho para mostrar sus debilidades, pero también para iluminarlo como objeto histórico. Rodríguez-Alcalá sigue los pasos de Romero en esta tarea y en la del desmenuzamiento de los supuestos del positivismo. Para csta segunda tarea expone la crítica de Romero a los dos grandes supuestos de la filosofía moderna: el racionalismo y el mecanicismo; y añade también la crítica al

3 Rodríguez-Alcalá ha iluminado los aspectos biográficos de Korn. En algunos casos obtuvo datos desconocidos; en otros, rcelaboró materiales existentes dándoles nueva vida, Buen ejemplo de lo primero es su ensayo "Apuntes para una biografía de Alejandro Korn" (Revista Iberoamencana, vol. XXIII, núm. 46, juldic. 1958) y, de lo segundo, su interesante estudio "Evocación de Alejandro Korn en Melchor Romero" (Asomante, Puerto Rico, vol. XIV, núm. 2, abril-junio 1958). Además, ha estudiado la personalidad del maestro argentino y su doctrina. Son muestras de ello "El socratismo de Alejandro Korn", "Razón y sentimiento en Alejandto Korn" - probablemente su mejor ensayo sobre el asunto-y "Alejandro Korn y el concepto de la historia"". Estos últimos escritos están reproducidos en su libro ya mencionado. 
empirismo. En cuanto al racionalismo, Romero ha hecho su crítica en general y, más que su crítica, su análisis. Este análisis, que se cuenta entre las páginas más penetrantes y lúcidas de Romero le importa a éste no sólo desde el punto de vista histórico, sino también en función de su personal doctrina filosófica. La "inmanentización" de la realidad que encuentra en el racionalismo es rechazada en su "metafísica de la trascendencia". En el parágrafo sobre la crítica al empirismo expone nuestro autor el análisis de Romero sobre la psicología empírica moderna, es decir, la psicología que arranca en Hume y culmina en Wundt y que Romero califica de "atomista", por estar calcada en sus fundamentos de la concepción moderna de la materia. Como es sabido, choca contra esta concepción la actitud "estructuralista" de Romero. Finalmente, Romero ha examinado los supuestos mecanicistas como uno de los rasgos fundamentales de la mentalidad moderna. Uno de los aspectos que más interesa destacar, como lo hace Rodríguez-Alcalá, es la "mecanización" de lo biológico por Darwin, en la que Romero ha insistido especialmente.

El propósito de los dos primetos capítulos, es decir, mostrar la crítica de Romero al positivismo, se logra plenamente. Pero en fuerza de destacar dicha crítica se supedita a ella la visión total que Romero tiene del pensamiento moderno. A nuestro entender, la compremsión de lo moderno tiene en Romero una estimable independencia, un valor en sí misma, en tanto que en el libro de Rodríguez-Alcalá se presenta, de hecho, demasiado vinculada a aquella crítica. El juicio de Romero sobre la trayectoria de la filosofía moderna es una interpretación de todo un período filosófico y de un momento de la cultura occidental. Dentro de ella, como un aspecto, tiene su lugar la comprensión - y, claro está, también la crítica-del positivismo, como lo tiene asimismo la de la filosofía contemporánea. Para Rodriguez-Alcalá, en cambio, la crítica al positivismo ocupa un lugar tan central que se convierte en el móvil que lleva a Romero a comprender el resto del proceso moderno. Por ello escribe: "Claro resulta, pues, el que Romero haya tomado la crítica del positivismo como punto de arranque para comprender la Edad Moderna, que es la que precede a la actual. La renovación ideológica no podía realizarse sin una elucidación de los errores del pasado para la persecución de las verdades del presente" (p. 52).

Finalmente, Rodríguez-Alcalá expone con acierto las relaciones que guarda la crítica de Romero al positivismo con el pensamiento del maestro argentino. "Su propia filosofía —escribe Alcalá-que rechaza el ra. 
cionalismo, que propugna un empirismo más amplio, que repulsa el atomismo y niega la validez metafísica del mecanicismo, no puede ser bien entendida sino a la luz de la crítica de lo que ella considera desvirtuación de la realidad, error inicial de una cosmovisión sobre que se fundan multitudes de errores'. (p. 52). Asimismo, señala los aspectos del análisis de Romero en que se hace justicia al positivismo, tanto a la doctrina europea como a sus expresiones argentinas.

En el capítulo titulado "El núcleo de la doctrina" expone el autor los conceptos de estructura, trascendencia y valor, considerándolos fundamentales en el pensamiento filosófico de Romero. Aunque el ca. pítulo se divide en tres apartados correspondientes a cada una de las nociones señaladas, de hecho se alude también a las ideas de intencionalidad y espítitu. De estos conceptos fundamentales que examina Rodríguez-Alcalá el más importante es el de trascendencia, y así.lo entiende nuestro crítico. Muy interesantes resultan las comparaciones que Rodríguez-Alcalá establece entre la esbozada "metafísica de la trascendencia" de Romero y las doctrinas de la emergent evolution, especialmente de Alexander. Creo que estas relaciones no habían sido señaladas anteriormente por los críticos de Romero. Este capítulo es central en el libro, pues en él se intenta apresar lo esencial -el "núcleo"- del pensamiento filosófico de Romero. No podríamos, en esta apreciación de conjunto, seguirlo en sus detalles; pero quetemos señalar dos características generales. La primera es que Rodríguez-Alcalá no adopta una actitud puramente descriptiva. Aunque sobre la base de un esfuerzo de comprensión, junto con las doctrinas que expone nos presenta las dudas o reservas que aquéllas le sugieren. Por obra de ello se ponen en evidencia, en algunos casos, aspectos no totalmente desarrollados hasta hoy por el filósofo argentino. La segunda observación es que en la exposición de conceptos fundamentales de la filosofía de Romero que realiza Rodríguez-Alcalá la idea de intencionalidad, con sus amplias consecuencias antropológicas, no pareciera estar suficientemente representada. No se trata de discutir ahora si los conceptos fundamentales escogidos por el autor son o no los más centrales, ${ }^{4}$ por cuanto todos están de tal modo relacionados que, como ocurre en la propia exposición de Rodríguez-Alcalá, no es posible hacer referencia a uno $\sin$ remitir a los otros. Nuestra observación apunta, so-

4 Por mi parte insinuaría que corresponden más bien al estado de la doctrina tal como ésta estaba expuesta en Papeles para una filosofía. En cambio, tal como ella puede apreciatse en Teoría del bombre, sugeritia que los conceptos fundamentales podrían ser: intencionalidad, espíritu y trascendencia. 
lamente, a una cuestión de proporciones en la doctrina total. En otras palabras, tenemos la impresión de que en este capítulo Rodríguez-Alcalá estuvo más preocupado por lo metafísico que por lo antropológico en el pensamiento de Romero, con lo cual creemos que no ha sido extraída de Teoria del hombre toda la sustancia posible.

En el capítulo siguicnte, basado principalmente en el contenido de Filosofía de la persona, Rodríguez-Alcalá expone "el concepto romeriano de persona en relación con el de libertad política" (p. 75). Su principal intención es "señalar el csfuerzo del pensador argentino de plantear los problemas prácticos de su tiempo en función de una metafísica del espíritu" (lug. cit.). Aquí encontramos la misma actitud indagadora del autor: cuestiona, pregunta, relaciona y, en algunos casos, llena con su personal interpretación los interrogantes que encuentra abiertos en los textos, o cree necesario abrir cuando estos textos no le resultan totalmente satis. factorios. Tampoco aquí podríamos seguir demasiado al detalle su labor, sino señalar algunos puntos principales. Dos cuestiones preocupan espccialmente a Rodríguez.Alcalá en la doctrina de la persona o el espíritu de Francisco Romero. Una es el carácter no sustancial de la persona. Rodríguez-Alcalá acerca esta idea a la concepción de Ortega del hombre como programa y se remonta al antecedente de la doctrina actualista del yo en Fichte. El carácter no sustancial de la persona y la discontinuidad de los actos espirituales le parecen a Rodríguez-Alcalá objetables en la doctrina de Romero. ¿Obra en el crítico el arraigado supuesto sustancialista? ¿Cómo explicar - pregunta- esa continuidad de la conducta como emergiendo de algo o como siendo algo, que es, además de discontinuo, insustancial?" (p. 82). ¿No estará la respuesta en Teoría del bombre, donde se afirma que el espíritu es un estrato superior de la realidad que se apoya sobre la psique (que seria el elemento de "continuidad") ? Además, ¿la precariedad de una forma de realidad le quita a ésta consistencia óntica y originalidad frente a otras formas de realidad? Por ejemplo, ¿el carácter inestable y frágil del equilibrio orgánico, en comparación con la estabilidad y solidez de lo inorgánico, podría privar a una célula de su peculiar realidad frente a una piedra? Lo que importa a Romero es que el espíritu tiene realidad propia, debido - digámoslo muy sencillamente-a su desinterés. Con ello se recorta un estrato nuevo de la realıdad, no importa cuál sea su continuidad o consistencia. En otras palabras, ser actualidad pura no le impide al espíritu ser, en un especial modo, realidad. La justa comprensión de este asunto no debe desvincularse de las teorías ontológicas de 
Nicolai Hartmann y de las del Scheler de El puesto del bombate en el cosmos.

Claro que a Romero no le importa solamente el espíritu como mero plano estático de la realidad total. Le interesa también en un sentido dinámico, como última y superior manifestación del impulso metafísico de la trascendencia. $Y$ con esto se relaciona la segunda preocupación de Rodríguez-Alcalá. En efecto, preocupa a nuestro crítico que Romero, filósofo de la persona, no quiera hablar de una "Persona Perfec. ta", y estima que esto podría considerarse una nueva "inmanentización". Asimismo, destaca Rodriguez-Alcalá que Romero, aunque opone a la tesis de Heidegger que reza: "el hombre es el ser para la muerte", la suya propia que afirma: "el hombre es el ser para el valor", no soluciona satisfactoriamente el problema de la inmortalidad. Pero ¿realmente se propuso Romero solucionar el problema de Dios y de la inmortalidad en el modo tradicional de entender esos problemas, que parece ser el modo en que se sitúa el crítico para analizar la doctrina del filósofo argentino? Nuestra opinión sería negativa. Rodríguez-Alcalá, recordando a Unamuno, pregunta: "Son los valores, en Romero, los conceptos de Dios y sustancia disfrazados?" Y responde: "Tal vez" (p. 89). Creo, por mi parte, que no hay tal disfraz, y que si Romero piensa en lo "divino" - si esta expresión pudiera emplearse- no lo concibe como exterior a la realidad sino como ínsito en ella. Aquí valdría la comparación con Alexander, que Rodríguez-Alcalá usó tan bien en otro caso. Pero como dice el propio autor, la obra de Romero no está completa y es el filósofo el encargado de decir la última palabra. ${ }^{5}$

El capítulo que ahora examinamos concluye con el examen -muy oportuno y no muy frecuentado anteriormente por otros críticos- de las consecuencias que se siguen de la filosofía de la persona de Romero sobre los problemas político-sociales y, en especial, sobre el tema de la libertad política. Partiendo Romero del hecho de que "no será posible en adelan. te escapar a la contraposición entre libertad y socialización", quiere aplicar

s Admito el carácter de construcción personal que tiene lo que afirmo, pero entiendo que en el pensamiento de Romero el elemento "divinidad" estaría en cierto modo en el hombre mismo, en tanto es parte de un proceso que apunta gradualmente a una perfección que, con especiales características, las teologías tradicionales ponen de una vez en el Ser Trascendente. Romero participaría, pues, en la forma especial de su "metafísica de la trascendencia", de la "doctrina del Dios que se hace". Las simpatias de Romero por esta idea me son evidentes, pero aquí lo apunto sólo como "hipótesis de trabajo". 
a esa problema la distinción entre individuo y puersona, que es esencial en su filosofía.

Finalmente, el último capítulo está dedicado al análisis de Romero sobre la crisis contemporánea. Hasta aquí, lo expuesto por Rodríguez-Alcalá se referia a dos principales temas: la crítica al positivismo, en primer lugar, y la metafísica de Romero, en segundo término. En otras palabras, el autor nos mostró primero la actitud de Romero ante el pasado filosófico inmediato, y luego nos expuso el personal contenido metafísico con que se llena esa actitud, puesto que no fue un mero polemizar, sino que se tradujo en una filosofía propia. Ahora bien, ¿qué relación guarda con estos dos momentos el examen del mundo contemporáneo? Nos lo dice Rodríguez-Alcalá: "Se ve ahora [a la luz de ese examen] con mayor claridad el significado de la labor de Romero. Su polémica antipositivista es un esfuerzo por deshacerse de la obra muerta del pasado; su metafísica, un replanteamiento de los problemas máximos del hombre, su mundo, su cultura. Su examen de la crisis, además de ser un intento de clarificar el presente, es también un intento de iluminar el camino del porvenir" (p. Ioo). Rodríguez-Alcalá expone con gran claridad las ideas de Romero sobre la crisis. Antes de comentarlas en detalle las resume nítidamente en breves párrafos: "Ni el hombre, ni su civilización, ni el occidente han fracasado. Tan sólo una etapa de la cultura occidental ha llegado a su fin, y por lo tanto, esa cultura ha entrado en crisis. Esa etapa es, como se ha indicado, la Edad Moderna". ( $\mathrm{P}$. IOI). Y tras presentar las opiniones en que Romero apoya esta conclusión, alude Rodríguez-Alcalá a las ideas de aquél sobre las grandes culturas (Occidente, China, India) y a la fe de Romero en el futuro del hombre occidental, que con tanta convicción y belleza está expresada en los párrafos finales de Teoría del bombre.

El libro se cierra con una bibliografía de escritos de y sobre Romero, y de otros materiales utilizados por el autor.

Intentando ahora una apreciación de conjunto de esta notable aportación de Rodríguez-Alcalá, queremos señalar en primer lugar que el libro participa, en oportuna medida, de dos modos de acceso al tema: el ensayo cálidamente humano, de aproximación simpática y que pone de relieve la significación espiritual de la obra analizada; y el estudio riguroso, con raíces bien orientadas en las fuentes que interpreta. Esto, unido a un interés vivo, que da a las observaciones del autor el tono de lo participado, y no sólo de lo visto desde fuera, otorga "personalidad" al libro. Coincidimos con Ferrater Mora cuando afirma, en el prólogo, que la 
obra de Rodríguez-Alcalá es "el primer trabajo a fondo y completo que se escribe sobre Francisco Romero". Nada de lo anterior puede comparársele, ni en amplitud ni en resultados. No quiere esto decir, sin embargo, que todos los aspectos de la obra filosófica de Romero hayan sido analizados. Pero esto no es contradictorio con lo anterior, si se atiende a la intención del autor. Rodríguez-Alcalá, nos parece, no se propuso escribir un libro "sistemático", donde todos los aspectos del pensamiento de Romero fueran detenidamente examinados, es decir, algo así como un mapa de su obra filosófica. Creemos que su intención fue señalar un tránsito, mostrar cómo el pensamiento de Romero es un pensamiento rector en una etapa que está reemplazando a otra, o, en otras palabras, cómo su pensamiento expresa su misión, la misión de reemplazar al positivismo y, consiguientemente, de ampliar el horizonte filosófico de la Argentina. Esta dimensión histórica es fundamental para el autor y en función de ella se organiza la exposición de las doctrinas. La intención puede darse por cumplida, y en ello reside lo más original del libro. Pero es riesgo inherente a toda visión en perspectiva la posibilidad de distorsionar en alguna medida la imagen, y así crecmos que ocurre aquí, en un determinado aspecto. Tal como aparece en el libro de Rodríguez-Alcalá, la función de Romero en el desplazamiento del positivismo en la Argentina resulta - sin que el autor se lo proponga- magnificada. La mención de Korn en la Introducción no será bastante para impedir que un lector desprevenido no acierte a distinguir las diferencias que hay entre ambos en lo que respecta a su relación con el ambiente positivista. Lo cierto es que cuando el pensamiento de Romero madura y empieza a pesar decisivamente en el ambiente filosófico argentino (para traducir este momento en una fecha podemos elegir el año I930), hace ya décadas que en la Argentina Korn y Alberini, por citar a los principales, vienen combatiendo con éxito al positivismo. Hoy se nos hace claro que Romero ha resultado ser, precisamente, la gran figura de la etapa que sigue a este combate, es decir, de la etapa de reemplazo propiamente dicho del positivismo, y no de polémica contra él, aunque en dicha polémica haya intervenido. Lo que Rodríguez-Alcalá nos dice sobre las críticas de Romero al positivismo es correcto; pero es la significación histórica de esas críticas en comparación con otras lo que no queda del todo precisada. Una mayor atención al contexto histórico en el que Romero estuvo inmerso hubicra permitido situarlo con mayor precisión en el proceso de combate y reemplazo del positivismo.

Esta salvedad no amengua los méritos antes reconocidos. Si el libro 
nos ha suscitado observaciones es porque él, a su vez, es tico en ellas y se presta al intercambio fructífero de opiniones. Lo que más nos importa destacar es que el autor ha elevado el nivel y abierto una etapa en el estudio de la obra de Romero. Cuando esta labor se continúe, por la preocupación del propio Rodríguez-Alcalá o de otros, este libro quedará más señalado todavía en su condición de punto de arranque y, me animo a predecir, seguirá ocupando un lugar destacado en la bibliografía sobre el gran maestro argentino, cuya significación filosófica y humana justifica largamente este esfucrzo y los que se hagan en el futuro.

Juan Carlos Torchia-Estrada

Unión Panamiericana.

Washington D.C., E.U.A. 
